\title{
Transmission Electron Microscope Observation of Charge Distribution on Insulating Thin Films by Hydro-carbon Deposition
}

\author{
Ken Harada ${ }^{1}$, Keiko Shimada ${ }^{1}$, Kodai Niitsu $^{1}$, Teiji Katsuta ${ }^{2}$, Teruaki Ohno ${ }^{3}$, and Daisuke Shindo ${ }^{1,4}$ \\ 1. CEMS, RIKEN (The Institute of Physical and Chemical Research), Hatoyama, Saitama, Japan \\ 2. Vacuum Device Inc., Mito, Ibaraki, Japan \\ 3. Tecnex Lab., Machida, Tokyo, Japan \\ 4. IMRAM, Tohoku University, Sendai, Miyagi, Japan
}

In transmission electron microscopes (TEMs), high energy incident electron beams onto specimens generate and induce many kinds of secondary electrons (SEs) in wide spatial directions and wide energy bands [1,2]. Equilibrium state of charge distribution on specimen-films depends on SEs scattered out from the specimen-films and absorbed on them. For conductive materials as specimens, electric charges due to SEs are grounded immediately, and the whole specimen area is neutralized. However, for insulating materials, such as organic materials and bio-materials, SEs accumulate and charge up, which causes damage and contamination on films. To detect charge distributions on insulating films, we applied a hydro-carbon (HC) replica method, which shows distribution of absorption and accumulation of positively or negatively ionized hydro-carbon molecules in residual gases in a TEM column [3, 4].

A high current density electron beam of about $0.5 \mathrm{~A} / \mathrm{m}^{2}$ at $75 \mathrm{kV}$ from a tungsten hairpin cathode was irradiated on $\mathrm{Si}_{3} \mathrm{~N}_{4}$ membranes (10 and $30 \mathrm{~nm}$ thick) for 2 and 3 hours, using H-7100 TEM (Hitachi High-Technologies Corp.). Then bright-field images of their hydro-carbon replicas were observed with 100-kV TEM (HF-3300S, Hitachi High-Technologies Corp.). Contrast of the image showed an equilibrium charge distribution during the electron beam irradiation.

Figure 1(a) shows a hydro-carbon replica image of the irradiation pattern on the 30-nm thick $\mathrm{Si}_{3} \mathrm{~N}_{4}$ membrane with a 500- $\mu \mathrm{m}$ square opening. The white spot at the center of the image is the beam irradiation point. The electron beam of about $2 \mu \mathrm{m}$ in diameter was irradiated near the corner of the $\mathrm{Si}$ substrate. The replica image composed of one radial straight line and two curves along the opening-edge of the Si substrate. We conjecture that SEs from the substrate are the source of these images: SEs from the $\mathrm{Si}_{3} \mathrm{~N}_{4}$ membrane (SE1 and SE2) excited other SEs at the Si substrate (SE3) and they were backscattered to the $\mathrm{Si}_{3} \mathrm{~N}_{4}$ membrane [1]. Figures 1(b) and (c) show schematic models of the cross-sectional structure of hydro-carbons, and scattered and absorbed SEs (SE1s, SE2s, and SE3s), respectively. In Fig. 1(b), charge distributions consist of three zones around the beam irradiation point: Zone I is positively charged by high-density irradiation electrons, Zone II is a neutralized zone by balancing the scattered and absorbed SEs, and Zone III is negatively charged by absorbed SEs. Since most of the hydro-carbon molecules are ionized in both polarities of charge, the neutral zone in the middle is thinnest.

To confirm the conjecture stated in Fig. 1(b) and (c), electron beams were irradiated on other $\mathrm{Si}_{3} \mathrm{~N}_{4}$ membranes of $10 \mathrm{~nm}$ thickness with a $25 \mu \mathrm{m}$ square opening. Replica images after two-hour irradiation clearly show the central white spot of irradiation point and four radial straight lines from each corner of the Si substrate opening, indicating the presence of SE3s from the substrate absorbed on the membrane. Furthermore, the diamond shaped pattern in Fig. 2(a) is slightly rotated counterclockwise. This is because the SE3s were azimuthally rotated by the magnetic field $\boldsymbol{H}_{\mathrm{obj}}$ of about $0.5 \mathrm{~A} / \mathrm{m}$ in the objective 
lens. Figures 2(b), (c), and (d) show replica images taken at different magnetic fields, 0.25, 0 and -0.5 $\mathrm{A} / \mathrm{m}$, respectively. The diamond shaped patterns are azimuthally rotated depending on the magnetic field. From these observation, we conclude that the image rotations are due to Larmor rotations of SE3s caused by the objective lens magnetic field [5].

\section{References:}

[1] L. Reimer, "Scanning Electron Microscopy”, (Springer, Berlin, 2013) p. 162.

[2] L. Reimer and H. Kohl, "Transmission Electron Microscopy", (Springer, Berlin, 2010) p. 484.

[3] J. T. Fourie, Optik 44 (1975) p. 111.

[4] J. J. Hren, Ultramicroscopy, 1 (1979) p. 375.

[5] The authors would like to thank A. Hisada of Hitachi Ltd., S. Nomoto of Hitachi High-Tech Fielding Corp., and K. Tamura and T. Yaguchi of Hitachi High-Technologies Corp. for their technical support.
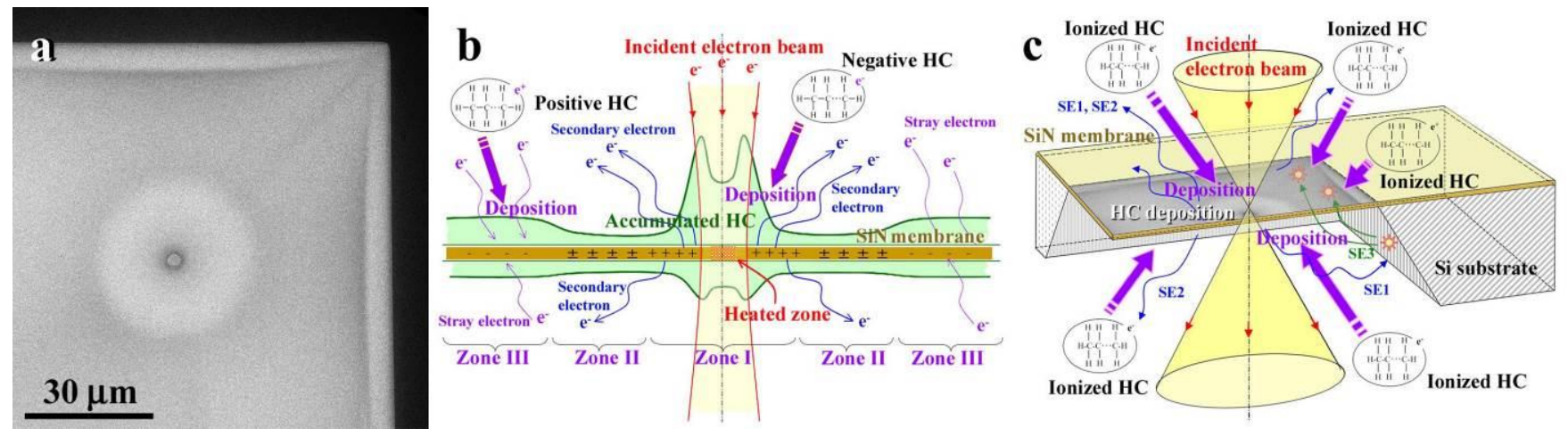

Figure 1. (a) Hydro-carbon (HC) replica image on $\mathrm{Si}_{3} \mathrm{~N}_{4}$ membrane near the corner of Si substrate after three-hour irradiation, (b) schematic model of the cross-sectional structure of accumulated hydrocarbons, and (c) schematic model of scattered and absorbed SEs, (SE1s, SE2s, and SE3s).
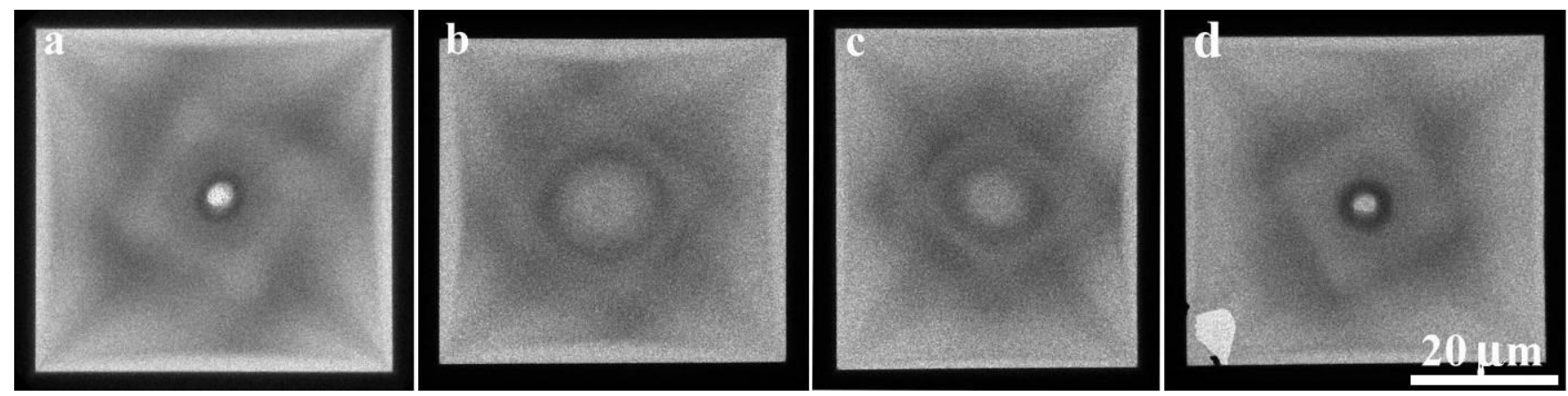

Figure 2. Hydro-carbon replica images depending on magnetic field $\boldsymbol{H}_{\mathrm{obj}}$ applied by objective lens, (a) $\boldsymbol{H}_{\mathrm{obj}}=0.5 \mathrm{~A} / \mathrm{m}$, (b) $\boldsymbol{H}_{\mathrm{obj}}=0.25 \mathrm{~A} / \mathrm{m}$, (c) $\boldsymbol{H}_{\mathrm{obj}}=0 \mathrm{~A} / \mathrm{m}$, and (d) $\boldsymbol{H}_{\mathrm{obj}}=-0.5 \mathrm{~A} / \mathrm{m}$. 